\title{
Inhibition of RAGE signaling through the intracellular delivery of inhibitor peptides by PEI cationization
}

\author{
ENDY WIDYA PUTRANTO ${ }^{1}$, HITOSHI MURATA ${ }^{1}$, KEN-ICHI YAMAMOTO $^{1}$, KEN KATAOKA ${ }^{2}$, \\ HIDENORI YAMADA $^{3}$, JUN-ICHIRO FUTAMI ${ }^{3}$, MASAKIYO SAKAGUCHI ${ }^{1}$ and NAM-HO HUH ${ }^{1}$
}

\author{
${ }^{1}$ Department of Cell Biology, Okayama University Graduate School of Medicine, Dentistry and Pharmaceutical Sciences, \\ Okayama 700-8558; ${ }^{2}$ Department of Life Science, Faculty of Science, Okayama University of Science, \\ Okayama 700-0005; ${ }^{3}$ Department of Medical Bioengineering Science, Okayama University \\ Graduate School of Natural Science and Biotechnology, Okayama 700-8530, Japan
}

Received May 15, 2013; Accepted July 26, 2013

DOI: $10.3892 / \mathrm{ijmm} .2013 .1467$

\begin{abstract}
The receptor for advanced glycation end products (RAGE) is a multi-ligand cell surface receptor and a member of the immunoglobulin superfamily. RAGE is involved in a wide range of inflammatory, degenerative and hyper-proliferative disorders which span over different organs by engaging diverse ligands, including advanced glycation end products, S100 family proteins, high-mobility group protein B1 (HMGB1) and amyloid $\beta$. We previously demonstrated that the cytoplasmic domain of RAGE is phosphorylated upon the binding of ligands, enabling the recruitment of two distinct pairs of adaptor proteins, Toll-interleukin 1 receptor domain-containing adaptor protein (TIRAP) and myeloid differentiation protein 88 (MyD88). This engagement allows the activation of downstream effector molecules, and thereby mediates a wide variety of cellular processes, such as inflammatory responses, apoptotic cell death, migration and cell growth. Therefore, inhibition of the binding of TIRAP to RAGE may abrogate intracellular signaling from ligand-activated RAGE. In the present study, we developed inhibitor peptides for RAGE signaling (RAGE-I) by mimicking the phosphorylatable cytosolic domain of RAGE. RAGE-I was efficiently delivered into the cells by polyethylenimine (PEI) cationization. We demonstrated that RAGE-I specifically bound to TIRAP and abrogated the activation of Cdc42 induced by ligand-activated RAGE. Furthermore, we
\end{abstract}

Correspondence to: Professor Nam-ho Huh, Department of Cell Biology, Okayama University Graduate School of Medicine, Dentistry and Pharmaceutical Sciences, 2-5-1 Shikata-cho, Kita-ku, Okayama 700-8558, Japan

E-mail: namu@md.okayama-u.ac.jp

Abbreviations: RAGE, receptor for advanced glycation end products; RAGE-I, inhibitor peptides for RAGE signaling; TIRAP, Toll-interleukin 1 receptor domain-containing adaptor protein; MyD88, myeloid differentiation protein 88; PEI, polyethylenimine

Key words: receptor for advanced glycation end products, Tollinterleukin 1 receptor domain-containing adaptor protein, cationization, S100B, cell death, cell migration were able to reduce neuronal cell death induced by an excess amount of S100B and to inhibit the migration and invasion of glioma cells in vitro. Our results indicate that RAGE-I provides a powerful tool for therapeutics to block RAGE-mediated multiple signaling.

\section{Introduction}

The receptor for advanced glycation end products (RAGE) belongs to the immunoglobulin superfamily of cell surface receptors. It comprises of three domains, an extracellular domain (with one V-type and two C-type domains), a transmembrane-spanning domain and a cytoplasmic domain (1). RAGE is activated by binding to a diverse repertoire of ligands (2). These ligands include advanced glycation end products (AGEs) (3), S100 family proteins (4-6), high-mobility group box 1 (HMGB1) $(5,7)$ and amyloid $\beta$ (A $\beta$ ) peptides (8). Activated RAGE triggers multiple intracellular pathways, such as the production of reactive oxygen species, the activation of p21ras, Erk1/2 (p44/p42) mitogen-activated protein kinases, p38 and stress-activated protein kinase (SAPK)/c-Jun N-terminal kinase (JNK) mitogen-activated protein kinases, rhoGTPases, phosphatidylinositol-3 kinase and Janus kinase (JAK)/signal transducers and activators of transcription (STAT), eventually leading to the activation of nuclear factor- $\mathrm{\kappa B}(\mathrm{NF}-\mathrm{\kappa B})$, activator protein-1 (AP-1) and STAT-3 (9-12).

RAGE is physiologically expressed in multiple tissues during embryonic development, but is mostly downregulated as cells reach homeostasis in adult life. Therefore, with the exception of the skin and lungs, RAGE expression is kept at low levels in the adult body $(13,14)$. The binding of ligands and the increased expression of RAGE potentially lead to inflammation-associated pathological conditions, such as those found in neurodegenerative diseases $(8,15,16)$, cardiovascular and renal diseases $(17,18)$, pulmonary diseases (19), diabetes and metabolic disorders $(20,21)$, as well as cancer (22). Under such conditions, NF- $\kappa B$ activated by RAGE signaling enhances the expression of RAGE, thus composing a self-sustaining positive feedback loop. Therefore, RAGE is one of the most promising targets for the development of therapeutic methods. 
Antagonistic RAGE peptides (23,24), RAGE-blocking antibodies (25) and ligand-binding drugs (26) have been examined in an effort to inhibit or abrogate RAGE signaling. These studies focused on the extracellular domain of RAGE and its various ligands. On the other hand, we have previously found that the short cytoplasmic domain of RAGE is phosphorylated at serine 391 (Ser391) by protein kinase C, zeta $(\mathrm{PKC} \zeta)$ upon ligand binding. The phosphorylated domain then recruits Toll-interleukin 1 receptor domain-containing adaptor protein (TIRAP), an adaptor protein for TLR2/4 (27), and transduces a signal to downstream molecules. Since the RAGE-TIRAP interaction is commonly induced by diverse ligands, such as AGEs, S100 proteins and HMGB1, an inhibitory tool for the interaction between RAGE and TIRAP may efficiently abrogate RAGE-mediated signaling regardless of ligand species. In the present study, we developed inhibitor decoy peptides for RAGE signaling (RAGE-I) by mimicking the phosphorylated cytosolic domain of RAGE. RAGE-I was cationized by polyethylenimine (PEI) for its efficient delivery into target cells, as previously described (28). We showed that RAGE-I, delivered intracellularly, bound to TIRAP and mitigated RAGE-mediated signaling. Neuronal cell death induced by an excess amount of S100B and the migration and invasion of glioma cells were suppressed by the application of RAGE-I in vitro. Our results indicate that RAGE-I provides a powerful therapeutic tool for the blocking of RAGE-mediated multiple signaling.

\section{Materials and methods}

Preparation of PEI-avidin and biotinylated RAGE-I. To efficiently deliver inhibitor peptides into cells, PEI-avidin was used as the vehicle as previously described (28). Avidin was coupled with PEI600 (both from Wako Chemicals, Osaka, Japan) by 1-ethyl-3-(3-dimethylaminopropyl) carbodiimide hydrochloride (EDC; Pierce, Rockford, IL, USA) [avidin $(2.5 \mathrm{mg} / \mathrm{ml})$ in PEI600-solution $(100 \mathrm{mg} / \mathrm{ml}, \mathrm{pH} 5.0)$ and $0.1 \mathrm{mg} / \mathrm{ml} \mathrm{EDC]} \mathrm{for} 16 \mathrm{~h}$ at room temperature. After the reaction, the solution was exhaustively dialyzed against PBS. Inhibitor peptides were designed to function as a decoy by binding to TIRAP, mimicking the cytoplasmic sequence of RAGE (387-395 amino acids). Ser391 was replaced with alanine [RAGE(A)-I, non-phosphorylatable] or with glutamic acid [RAGE(E)-I, phosphorylation mimic] (Fig. 1). The peptides were biotinylated under conventional conditions. In SS-RAGE(E)-I, a disulfide bond was introduced between the peptide and biotin to facilitate the release of RAGE(E)-I from the PEI-avidin vehicle under intracellular reducing conditions as previously described $(29,30)$.

Cells, chemicals and antibodies. SH-SY5Y human neuroblastoma, U-87MG human glioblastoma, B16-BL6 mouse melanoma and HEK293 human embryonic kidney cells were cultured in D/F medium supplemented with $10 \%$ fetal bovine serum (both from Life Technologies, Carlsbad, CA, USA).

The antibodies used were as follows: goat polyclonal antibody against glutathione S-transferase (GST; GE Healthcare, Waukesha, WI, USA); mouse monoclonal antibody against Myc and HRP-labeled anti-mouse secondary antibody (Cell Signaling Technologies, Beverly, MA, USA). The Rac/Cdc42
Activation Assay kit was purchased from Millipore (Billerica, MA, USA). Endo-Porter was purchased from Gene Tools, LLC (Philomath, OR, USA).

Purification of recombinant proteins. GST, GST-TIRAP, GST-myeloid differentiation protein 88 (MyD88), GST-thyroid receptor activator molecule (TRAM) and GST-S100B proteins were purified from the bacterial lysates of BL21 competent cells transformed with pGEX6P1 vectors containing each cDNA using glutathione sepharose beads (GE Healthcare) according to the standard procedures outlined by the manufacturer. For the purification of S100B, GST-S100B was incubated with PreScission protease (1 U/100 $\mu \mathrm{g}$ of GST-S100B; GE Healthcare) for the cleavage of GST. GST was removed using glutathione sepharose beads. The purity of the proteins was determined by SDS-PAGE.

Pull-down assay and western blot analysis. Biotinylated peptides were incubated with GST-proteins or cell lysates for $2 \mathrm{~h}$. The pull-down of biotinylated peptide-protein complexes was carried out using streptavidin-coupled Dynabeads (Life Technologies).

Western blot analysis was performed under conventional conditions after lysing the cells with M-PER Mammalian Protein Extraction Reagent (Thermo Scientific, Lafayette, CO, USA) with PhosphoSTOP (Roche Applied Science, Indianapolis, IN, USA).

Apoptosis assay, migration assay and invasion assay. For apoptosis assay, the SH-SY5Y cells were cultured with $\mathrm{D} / \mathrm{F}-0.5 \% \mathrm{FBS}$ for $24 \mathrm{~h}$ prior to incubation with GST or $\mathrm{S} 100 \mathrm{~B}$ at $0-10 \mu \mathrm{M}$ for $24 \mathrm{~h}$. Apoptotic cells were identified after staining with Hoechst 33342 (Life Technologies) for $30 \mathrm{~min}$. For migration assay, 10,000 U-87MG cells incubated with inhibitor peptides in advance were inoculated into the top wells of Boyden chambers (pore size, $8 \mu \mathrm{m}$; BD Falcon, Franklin Lakes, NJ, USA). The filters were stained after incubation for $8 \mathrm{~h}$ and the migrated cells were counted. For invasion assay, 25,000 U-87MG cells incubated with inhibitor peptides in advance were inoculated into the top wells of Boyden chambers pre-coated with growth factor-reduced Matrigel (Life Technologies). The filters were stained after incubation for $8 \mathrm{~h}$ and invaded cells were counted.

Cell counting and cell growth assay. Cells were counted using a haemocytometer after staining with $0.5 \%$ trypan blue. Each experiment was repeated at least three times. For cell growth assay, the CellTiter-Glo Luminescent Cell Viability Assay system (Promega Biosciences, San Luis Obispo, CA, USA) was used. The experiment was carried out according to the manufacturer's instructions.

Rac/Cdc42 activation assay. The experiment was performed using the Rac1/Cdc42 Activation kit (Millipore) according to the manufacturer's instructions. U-87MG cells were treated with PEI-avidin/biotin-inhibitor peptides for $24 \mathrm{~h}$. The activation of Cdc42 (GTP bound form) was determined by pull-down assay using GST-PAK-1 (containing 67-150 amino acids) and glutathione sepharose beads followed by western blot analysis. 


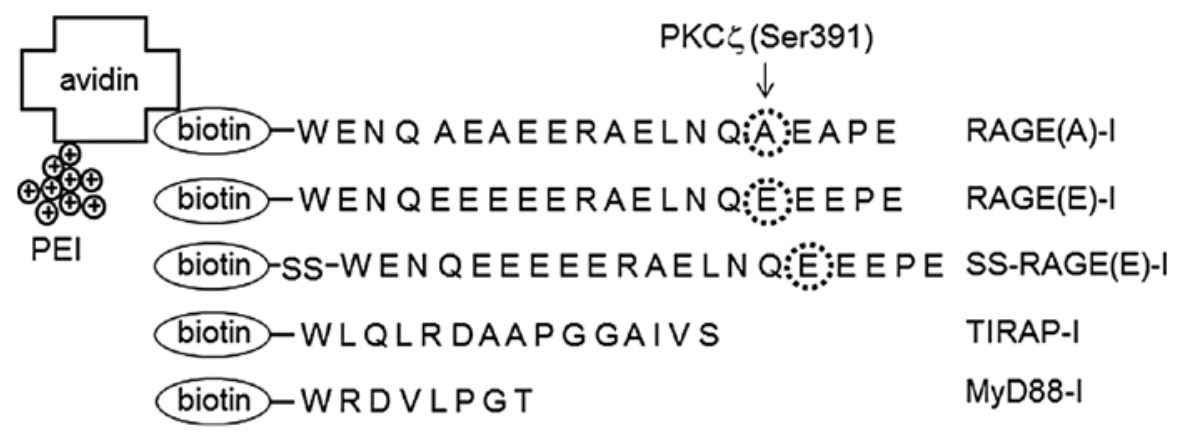

Figure 1. Constructs of receptor for advanced glycation end products (RAGE) inhibitor peptides (RAGE-I). Cytoplasmic region of RAGE (387-395 amino acids) interacting with the adaptor protein Toll-interleukin 1 receptor domain-containing adaptor protein (TIRAP) was used as a template for RAGE inhibitor peptides. Ligand-induced phosphorylation site of Ser391 was replaced either with non-phosphorylatable alanine [RAGE(A)-I] or with phosphorylation-mimic glutamic acid [RAGE(E)-I]. The inhibitor peptides were biotinylated for intracellular delivery using a polyethylenimine (PEI)-avidin vehicle. SS-RAGE(E)-I contains a disulfide bond between biotin and the peptide. SS-RAGE(E)-I can be released from the vesicle under intracellular reducing conditions. Inhibitor peptides for TIRAP (TIRAP-I) and myeloid differentiation protein 88 (MyD88)-I are known to function as decoys by binding to the TIR domain.

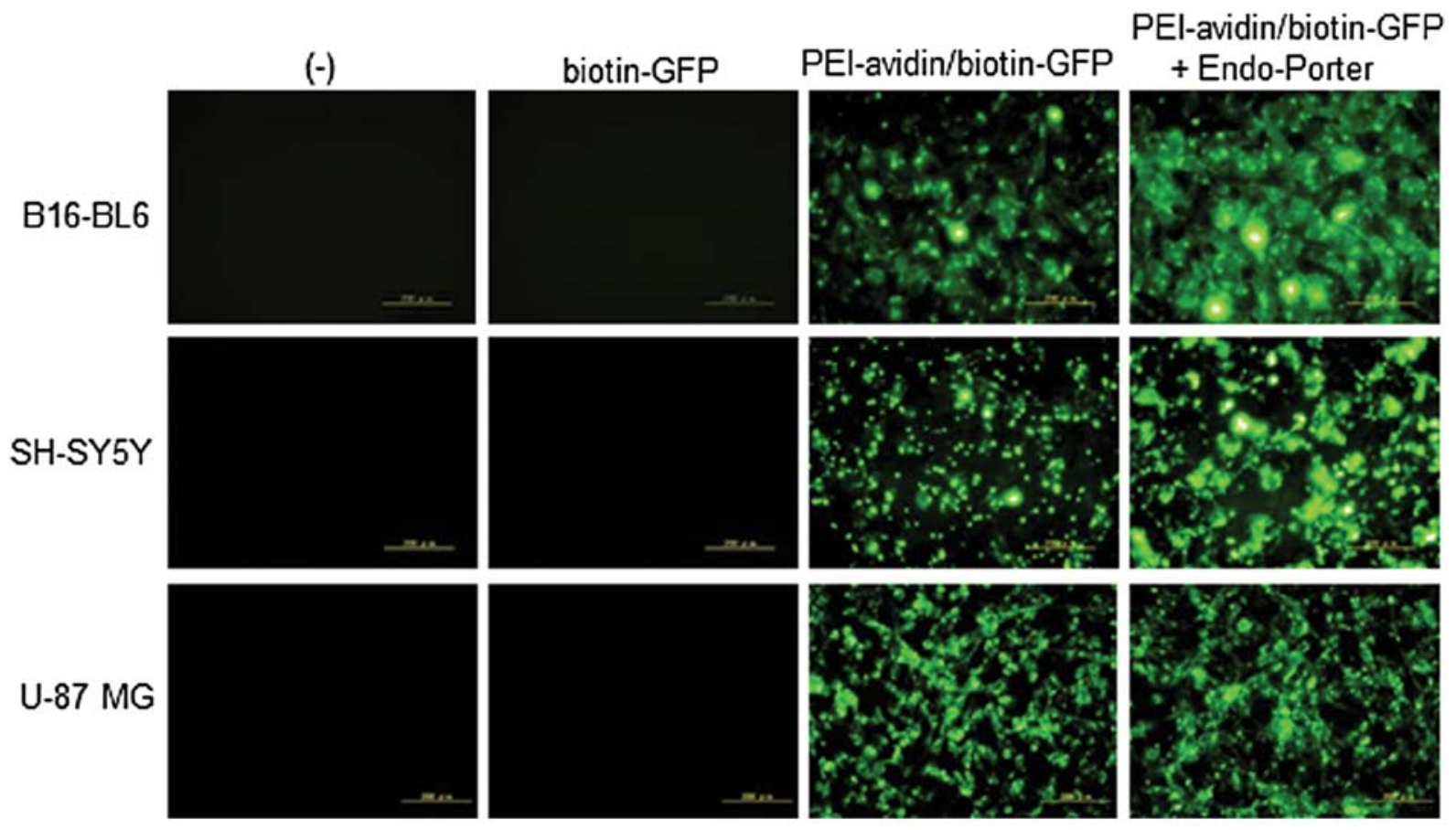

Figure 2. Intracellular delivery of biotinylated GFP. B16-BL6, SH-SY5Y and U-87MG cells were incubated with $1 \mu$ M biotin-GFP and/or PEI-avidin for $6 \mathrm{~h}$. Endo-Porter was added to the medium immediately after the addition of the proteins. The cells were washed thoroughly before being observed under a fluorescence microscope. Bar scale, $200 \mu \mathrm{m}$.

Statistical analysis. Prior to statistical analysis, each experiment was repeated at least three times. The results are expressed as the means \pm SD. For comparison, analysis of variance (ANOVA) was used. If ANOVA showed significant differences, the Bonferroni procedure was used as a post hoc test. P-values $<0.05$ were considered to indicate statistically significant differences.

\section{Results}

Preparation and intracellular delivery of RAGE-I. In our previous study, we showed that the phosphorylation of Ser391 of the intracellular domain of RAGE by PKC $\zeta$ was critical for recruiting the downstream signal transducer, TIRAP (27). In this study, we therefore designed decoy peptides covering the serine residue, i.e., 387-395 of RAGE. As shown in Fig. 1, we prepared three peptides, RAGE(A)-I, RAGE(E)-I and SS-RAGE(E)-I (see Materials and methods). Two inhibitor peptides for TIRAP (TIRAP-I) and MyD88 (MyD88-I), which were already reported to function as a decoy for TLR $2 / 4$ by binding to the TIR domain of TIRAP and MyD88 $(31,32)$, were included for comparison. The peptides were biotinylated for intracellular delivery using avidin conjugated with PEI-avidin. PEI cationization is a powerful tool used to deliver a protein or peptide into cells (28). To confirm the efficient transduction of biotinylated molecules by PEI-avidin, we examined the intracellular delivery of biotin-GFP using B16-BL6 mouse melanoma, SH-SY5Y human neuroblastoma and U-87MG human glioblastoma cells. As shown in Fig. 2, the PEI-avidin/biotin-GFP complex, but not the biotin-GFP alone 
A

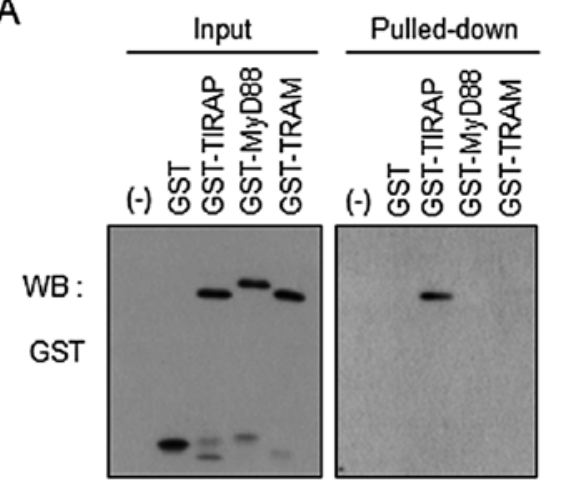

B

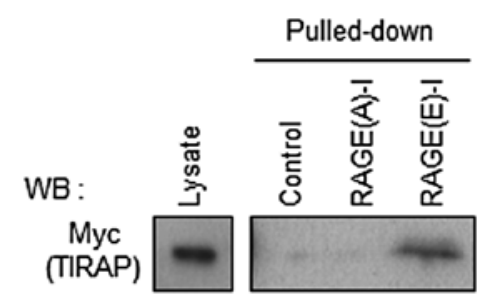

Figure 3. Binding of inhibitor peptides for receptor for advanced glycation end products (RAGE) signaling (RAGE-I) to Toll-interleukin 1 receptor domain-containing adaptor protein (TIRAP). (A) Biotinylated RAGE(E)-I was incubated with purified GST-fused proteins in vitro and was pulled-down using streptavidin-beads. Binding of RAGE(E)-I to TIRAP was detected by western blot analysis. (B) The lysates of HEK293 cells expressing Myc-TIRAP were incubated with biotinylated RAGE(A)-I or biotinylated RAGE(E)-I for $2 \mathrm{~h}$. The binding of RAGE inhibitor peptides to TIRAP was determined by pull-down with streptavidin-beads followed by western blot analysis (WB).

was delivered efficiently into the cells. Cationized molecules are known to incorporate into cells through the macropinocytosis pathway (33) and Endo-Porter is known to enhance the release of incorporated molecules from the endosome to the cytoplasm (30). The fluorescence from intracellular GFP was intensified and showed a more diffused pattern in the cytoplasm by application of the cells with Endo-Porter (Fig. 2). Therefore, we used the combination of PEI-avidin and Endo-Porter for the intracellular delivery in the following experiments.

Direct binding of RAGE(E)-I to TIRAP. We prepared GST-fused TIRAP and other adaptor proteins of Toll-like receptors, MyD88 and TRAM, and incubated them with RAGE(E)-I in vitro. We detected only TIRAP in the pulled-down fraction of RAGE(E)-I, indicating the direct binding of the inhibitor peptide to TIRAP, but not to MyD88 and TRAM (Fig. 3A). We then examined the significance of the phosphorylation site at Ser391 of RAGE-I for interaction with TIRAP. Biotinylated RAGE-I was incubated with cell lysates expressing Myc-TIRAP, pulled-down with streptavidin beads, and analyzed by western blot analysis. As shown in Fig. 3B, phosphorylation-mimic RAGE(E)-I strongly interacted with TIRAP, whereas non-phosphorylatable RAGE(A)-I did not show any appreciable binding with TIRAP. These results indicate that RAGE(E)-I is a promising tool for interfering with RAGE signaling through a decoy function for TIRAP.

RAGE-Iprotects cell death induced by S100B. Huttunen et al (5) reported that relatively high concentrations $(5 \mu \mathrm{M})$ of S100B induced apoptosis in neuronal cells. In this study, after confirming the cytotoxic effects of $10 \mu \mathrm{M}$ S100B on SH-SY5Y cells (Fig. 4B), we examined whether the prophylactic application of RAGE-I reduces S100B-induced cytotoxicity in SH-SY5Y cells. We highly purified recombinant human S100B protein (Fig. 4A, lane 7) and treated the cells with the protein. By monitoring nuclear shrinkage with Hoechst staining, an assessment of apoptotic cell death, we found that the excess amount of S100B $(10 \mu \mathrm{M})$ induced an increase in apoptotic cell death (Fig. 4B). By contrast, the prophylactic addition of RAGE(E)-I, SS-RAGE(E)-I, TIRAP-I and MyD88-I, but not RAGE(A)-I attenuated the apoptotic ratio (Fig. 4C). SS-RAGE(E)-I showed almost the same effect in comparison with TIRAP-I and MyD88-I. These results indicate that the signaling from RAGE-TIRAP interaction plays a critical role in the induction of S100B-mediated cell death and that this may be prevented by pre-treatment of the cells with RAGE inhibitor peptides.

RAGE-I inhibits cell migration and invasion, but not cell growth. It is known that the activation of RAGE results in enhanced migration and invasion of various types of cells $(34,35)$. Therefore, we also investigated whether RAGE-I inhibits the migration and invasion of U-87MG glioblastoma cells in vitro. When the cells were treated with RAGE-I, the migration and invasion of the cells were significantly suppressed (Fig. 5A and B). In addition, these results correlated with the inactivation of Cdc42 (Fig. 5C), which is closely associated with RAGE-mediated cell migration $(36,37)$. A higher suppression was observed in the SS-RAGE(E)-I-treated group compared with the RAGE(E)-I-treated group (Fig. 5A-C). This possibly occurred as RAGE(E)-I was released from biotin-PEI-avidin and more efficiently captured endogenous TIRAP. Under similar conditions, the growth of U-87MG cells was not significantly affected as assayed by determining the intracellular adenosine triphosphate (ATP) content (Fig. 5D), or by counting the number of cells (Table I). These results indicate that RAGE-I effectively abrogates the downstream signaling of ligand-activated RAGE in glioblastoma cells in terms of migration and invasion.

\section{Discussion}

RAGE plays pivotal roles in a variety of physiological contexts, including early development. On the other hand, the aberrant hyperfunctional state of RAGE due to the overexpression of RAGE and/or the overstimulation of RAGE by ligands is involved in a variety of pathological conditions, including diabetic syndrome, cancer and neurodegenerative diseases. Thus, interfering with RAGE function is considered a potent therapeutic measure. Abe et al (25) reported that RAGE was 

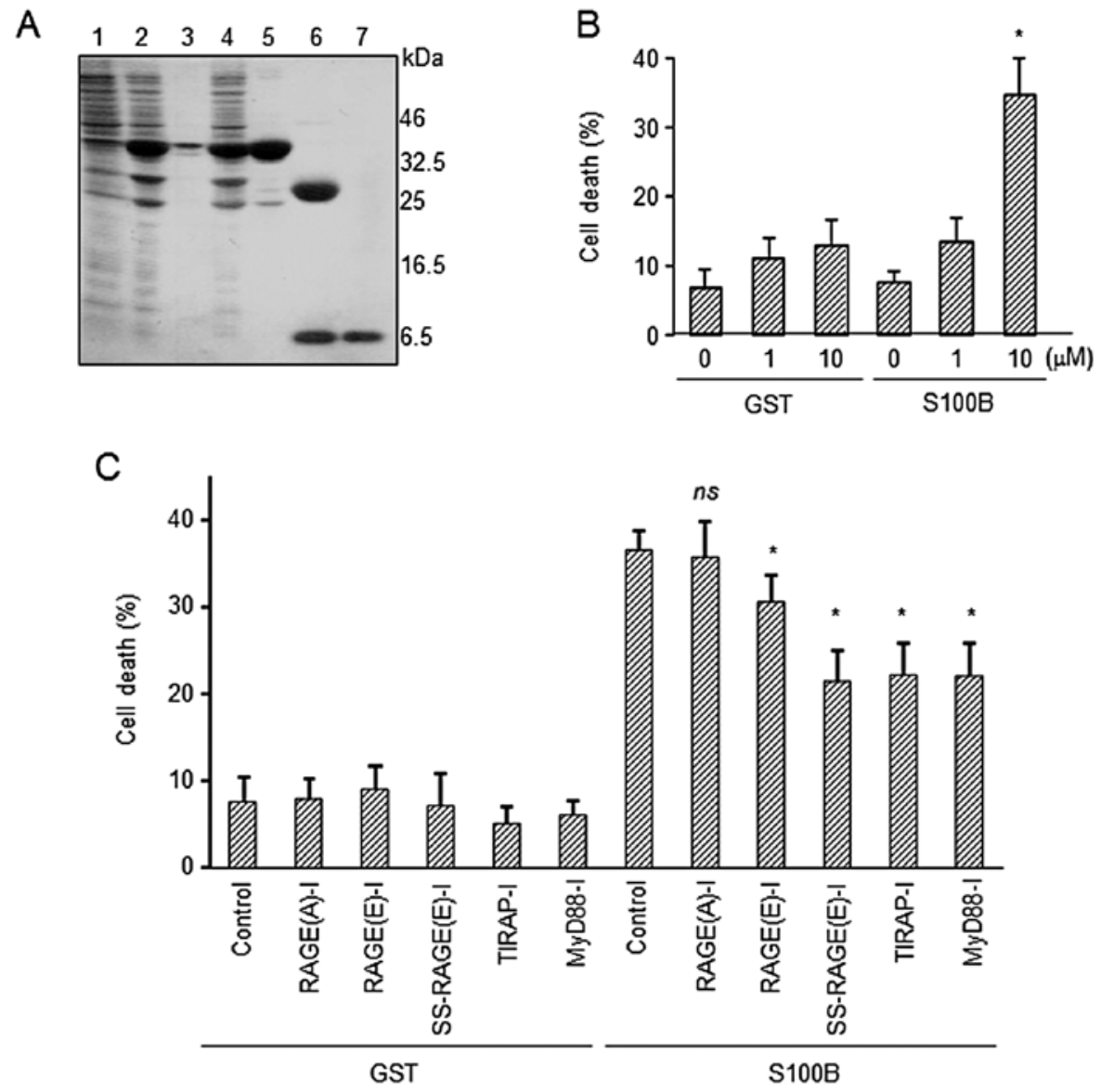

Figure 4. Mitigation of S100B-related cell death by inhibitor peptides for receptor for advanced glycation end products (RAGE) signaling (RAGE-I). (A) Expression and purification of S100B from E. coli. The purity of S100B was confirmed by SDS-PAGE. Lane 1, E. coli lysates without isopropyl $\beta$-D-1thiogalactopyranoside (IPTG) induction; lane 2, induction of GST-S100B with IPTG; lane 3, precipitates after lysis; lane 4, supernatants after lysis; lane 5, GST-S100B after purification with Sephadex 4B-column; lane 6, cleavage of GST from S100B by PreScission protease; lane 7, purified S100B. (B) Induction of apoptosis by S100B. SH-SY5Y cells were incubated with control GST or S100B for $24 \mathrm{~h}$. Apoptotic cells were identified after staining with Hoechst 33342 . "P $<0.05$, significantly different from the control group. (C) Polyethylenimine (PEI)-avidin/biotin-inhibitor peptides ( $1 \mu \mathrm{M}$ ) were added to the SH-SY5Y cells for $12 \mathrm{~h}$ prior to treatment with GST or S100B $(10 \mu \mathrm{M}, 24 \mathrm{~h})$. Apoptotic cells were identified after staining with Hoechst 33342 . Statistically significant differences were determined by comparing with the cells without S100B. "P<0.05, significantly different from the control group with S100B; ns, not significant.

Table I. Treatment with inhibitor peptides did not affect the growth of U-87MG cells.

\begin{tabular}{lc}
\hline Groups & No. of cells $\left(\times 10^{5}\right)$ \\
\hline Control & $10.9 \pm 0.8$ \\
RAGE(A)-I & $10.7 \pm 0.5$ \\
RAGE(E)-I & $10.5 \pm 0.5$ \\
SS-RAGE(E)-I & $10.8 \pm 0.7$ \\
TIRAP-I & $10.9 \pm 0.8$ \\
MyD88-I & $10.7 \pm 0.7$ \\
\hline
\end{tabular}

RAGE, receptor for advanced glycation end products; RAGE-I, inhibitor peptides for RAGE signaling; TIRAP, Toll-interleukin 1 receptor domain-containing adaptor protein; MyD88, myeloid differentiation protein 88 .

overexpressed in human melanoma cells and that AGE stimulated the growth and migration of the cells. They showed that the application of an antibody against the extracellular domain of RAGE suppressed the growth of melanoma cells in vitro and in vivo. Huttunen et al (23) demonstrated that a C-terminal motif of amphoterin (150-183 amino acids) bound to RAGE and inhibited the migration and invasion of human fibrosarcoma cells. Arumugam et al (24) prepared S100P-derived small peptides. The peptide inhibited the interaction of S100P, S100A4 and HMGB-1 with RAGE and abrogated the growth of pancreatic tumors and glioma. In Alzheimer's disease (AD), the $\mathrm{A} \beta$ peptide accumulates in plaque in the brain. RAGE mediates $A \beta$-induced perturbations in cerebral vessels, neurons and microglia in AD. Deane et al (38) identified a high-affinity RAGE-specific inhibitor (FPS-ZM1) that blocked $A \beta$ binding to the $\mathrm{V}$ domain of RAGE and inhibited $\mathrm{A} \beta 40$ - and $\mathrm{A} \beta 42$-induced cellular stress in RAGE-expressing cells in vitro and in the mouse brain in vivo.

These studies focused on the extracellular domain of RAGE and its various ligands. On the other hand, we focused on the intracellular domain of RAGE based on our previous findings of RAGE-TIRAP interaction (27). Inhibitor peptides were designed by mimicking the phosphorylated cytosolic domain of RAGE. Since the RAGE-TIRAP interaction is a common converged event for diverse ligands such as AGE, S100 proteins and HMGB1, we hypothesized that an inhibi- 
A

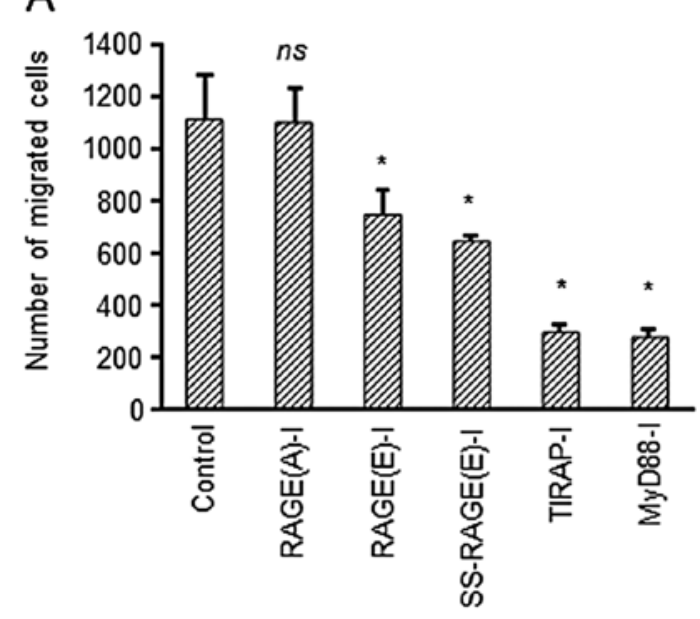

C

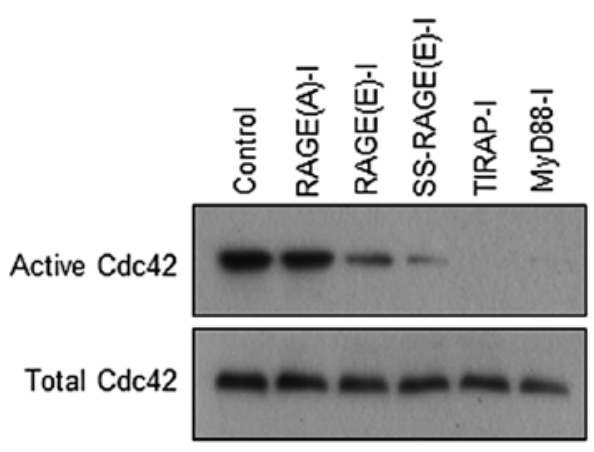

B
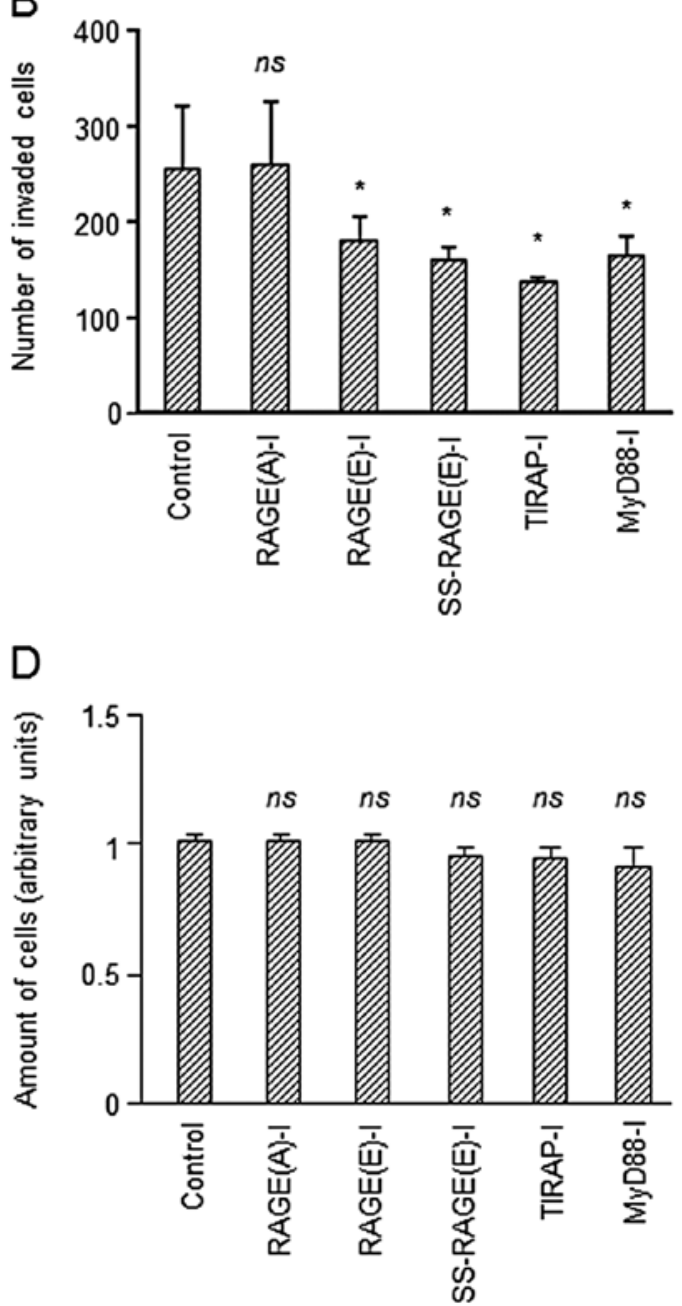

Figure 5. Inhibitor peptides for receptor for advanced glycation end products (RAGE) signaling (RAGE-I) suppress migration and invasion of glioma cells but not proliferation. $\mathrm{U}-87 \mathrm{MG}$ cells were incubated with $1 \mu \mathrm{M}$ polyethylenimine (PEI)-avidin/biotin-inhibitor peptides for $24 \mathrm{~h}$ for the (A) migration and (B) invasion assays and (C) determination of active Cdc42 (GTP bound form). (D) U-87MG cells were incubated with $1 \mu \mathrm{M}$ PEI-avidin/biotin-inhibitor peptides for $24 \mathrm{~h}$. The level of ATP was determined using CellTiter-Glo assay. ${ }^{*} \mathrm{P}<0.05$, significantly different from the control group; ns, not significant.

tory tool for the interaction between RAGE and TIRAP may efficiently abrogate RAGE-mediated signaling regardless of ligand species.

RAGE has been demonstrated to play a significant role in the development of several degenerative and inflammation-related diseases $(8,15,17-22)$. In the present study, we monitored cellular behavior related with two pathological conditions, neuronal cell death and the migration and invasion of cancer cells. Griffin et al (39) and Mrak and Griffinbc (40) demonstrated that S100B contributes to the progression of AD. In the present study, we demonstrated that the addition of S100B induced the apoptosis of SH-SY5Y cells and that the pre-treatment of the cells with RAGE inhibitor peptides resulted in the mitigation of apoptosis. The decrease in apoptosis suggests that RAGE-TIRAP interaction is one of the targets for the prevention of neurodegeneration. In cancer cells, cell migration and invasion are considered, at least in part, to occur due to the hyperfunction of RAGE (25). Since the high expression of RAGE and its ligands have previously been found in many cancer cases (22), in this study, we tried to inhibit cell migration and invasion by RAGE-I using U-87MG human glioblastoma cells. As expected, the treatment of U-87MG cells with RAGE-I resulted in the suppression of migration and invasion. The reduced migration and invasion appeared not be due to cytotoxicity as the cell growth was not affected (Table I) (Fig. 5D).

Protein transduction (PTD) sequences such as TAT, polyarginine and antennapedia are widely used for the intracellular delivery of proteins and peptides (33). Although the inhibitor peptides for TIRAP and MyD88 conjugated with the PTD sequence (Imgenex) are available, the recommended concentration for application is $\sim 100 \mu \mathrm{M}$. The necessity for high concentrations of the agents hampers the practical application of these peptides for future clinical, as well as experimental use. We demonstrated that PEI-avidin delivers biotinylated peptides at $1 \mu \mathrm{M}$ into cells and that the delivered peptides can function sufficiently. The addition of disulfide bonds to facilitate the release of the peptide from PEI-avidin under intracellular reducing conditions enhanced the efficiency of the inhibitor peptides. These data indicate that our approach provides a promising tool, not only for the analysis of the etiology of RAGE-related disorders, but also for the development of therapeutic measures against such diseases. 


\section{Acknowledgements}

The present study was supported in part by grants (AS242Z01065Q) from the Japan Science and Technology Agency (to H.M.), from the Ministry of Health, Labor and Welfare (Research for Intractable Diseases; to N.H.), from the Ministry of Education, Culture, Sports, Science and Technology of Japan (Grant-in-Aid for Scientific Research on Innovation Areas; to M.S.), from The Naito Foundation (to M.S.), from The Research Foundation for Pharmaceutical Sciences (to M.S.) and from The Ichiro Kanehara Foundation (to M.S.).

\section{References}

1. Neeper M, Schmidt AM, Brett J, et al: Cloning and expression of a cell surface receptor for advanced glycosylation end products of proteins. J Biol Chem 267: 14998-15004, 1992.

2. Schmidt AM, Yan SD, Yan SF and Stern DM: The biology of the receptor for advanced glycation end products and its ligands. Biochim Biophys Acta 1498: 99-111, 2000.

3. Schmidt AM, Mora R, Cao R, et al: The endothelial cell binding site for advanced glycation end products consists of a complex: an integral membrane protein and a lactoferrin-like polypeptide. J Biol Chem 269: 9882-9888, 1994.

4. Hofmann MA, Drury S, Fu C, et al: RAGE mediates a novel proinflammatory axis: a central cell surface receptor for S100/ calgranulin polypeptides. Cell 97: 889-901, 1999.

5. Huttunen HJ, Kuja-Panula J, Sorci G, Agneletti AL, Donato R and Rauvala $\mathrm{H}$ : Coregulation of neurite outgrowth and cell survival by amphoterin and S100 proteins through receptor for advanced glycation end products (RAGE) activation. J Biol Chem 275: 40096-40105, 2000.

6. Leclerc E, Fritz G, Weibel M, Heizmann CW and Galichet A: S100B and S100A6 differentially modulate cell survival by interacting with distinct RAGE (receptor for advanced glycation end products) immunoglobulin domains. J Biol Chem 282: 31317-31331, 2007.

7. Li J, Qu X and Schmidt AM: Sp1-binding elements in the promoter of RAGE are essential for amphoterin-mediated gene expression in cultured neuroblastoma cells. J Biol Chem 273: 30870-30878, 1998.

8. Yan SD, Chen X, Fu J, et al: RAGE and amyloid-beta peptide neurotoxicity in Alzheimer's disease. Nature 382: 685-691, 1996.

9. Huang JS, Guh JY, Chen HC, Hung WC, Lai YH and Chuang LY: Role of receptor for advanced glycation end-product (RAGE) and the JAK/STAT-signaling pathway in AGE-induced collagen production in NRK-49F cells. J Cell Biochem 81: 102-113, 2001.

10. Yeh CH, Sturgis L, Haidacher J, et al: Requirement for $\mathrm{p} 38$ and p44/p42 mitogen-activated protein kinases in RAGE-mediated nuclear factor-kappaB transcriptional activation and cytokine secretion. Diabetes 50: 1495-1504, 2001.

11. Lander HM, Tauras JM, Ogiste JS, Hori O, Moss RA and Schmidt AM: Activation of the receptor for advanced glycation end products triggers a p21(ras)-dependent mitogen-activated protein kinase pathway regulated by oxidant stress. J Biol Chem 272: 17810-17814, 1997.

12. Huttunen HJ, Kuja-Panula $\mathbf{J}$ and Rauvala $\mathrm{H}$ : Receptor for advanced glycation end products (RAGE) signaling induces CREB-dependent chromogranin expression during neuronal differentiation. J Biol Chem 277: 38635-38646, 2002.

13. Brett J, Schmidt AM, Yan SD, et al: Survey of the distribution of a newly characterized receptor for advanced glycation end products in tissues. Am J Pathol 143: 1699-1712, 1993.

14. Beccafico S, Riuzzi F, Puglielli C, et al: Human muscle satellite cells show age-related differential expression of S100B protein and RAGE. Age 33: 523-541, 2011.

15. Srikanth V, Maczurek A, Phan T, et al: Advanced glycation endproducts and their receptor RAGE in Alzheimer's disease. Neurobiol Aging 32: 763-777, 2011.

16. Maczurek A, Shanmugam K and Münch G: Inflammation and the redox-sensitive AGE-RAGE pathway as a therapeutic target in Alzheimer's disease. Ann NY Acad Sci 1126: 147-151, 2008.

17. Koyama $\mathrm{H}$ and Nishizawa $\mathrm{Y}$ : AGEs/RAGE in CKD: irreversible metabolic memory road toward CVD? Eur J Clin Invest 40: 623-635, 2010.
18. Reiniger N, Lau K, McCalla D, et al: Deletion of the receptor for advanced glycation end products reduces glomerulosclerosis and preserves renal function in the diabetic OVE26 mouse. Diabetes 59: 2043-2054, 2010.

19. Wu L, Ma L, Nicholson LF and Black PN: Advanced glycation end products and its receptor (RAGE) are increased in patients with COPD. Respir Med 105: 329-336, 2011.

20. Ramasamy R, Yan SF and Schmidt AM: Receptor for AGE (RAGE): signaling mechanisms in the pathogenesis of diabetes and its complications. Ann NY Acad Sci 1243: 88-102, 2011.

21. Su XD, Li SS, Tian YQ, Zhang ZY, Zhang GZ and Wang LX: Elevated serum levels of advanced glycation end products and their monocyte receptors in patients with type 2 diabetes. Arch Med Res 42: 596-601, 2011

22. Leclerc E, Heizmann CW and Vetter SW: RAGE and S100 protein transcription levels are highly variable in human melanoma tumors and cells. Gen Physiol Biophys 28: F65-F75, 2009.

23. Huttunen HJ, Fages C, Kuja-Panula J, Ridley AJ and Rauvala H: Receptor for advanced glycation end products-binding COOH-terminal motif of amphoterin inhibits invasive migration and metastasis. Cancer Res 62: 4805-4811, 2002.

24. Arumugam T, Ramachandran V, Gomez SB, Schmidt AM and Logsdon CD: S100P-derived RAGE antagonistic peptide reduces tumor growth and metastasis. Clin Cancer Res 18: 4356-4364, 2012.

25. Abe R, Shimizu T, Sugawara H, et al: Regulation of human melanoma growth and metastasis by AGE-AGE receptor interactions. J Invest Dermatol 122: 461-467, 2004.

26. Arumugam T, Ramachandran V and Logsdon CD: Effect of cromolyn on S100P interactions with RAGE and pancreatic cancer growth and invasion in mouse models. J Natl Cancer Inst 98: 1806-1818, 2006.

27. Sakaguchi M, Murata H, Yamamoto K, et al: TIRAP, an adaptor protein for TLR2/4, transduces a signal from RAGE phosphorylated upon ligand binding. PLoS One 6: e23132, 2011.

28. Kitazoe M, Murata H, Futami J, et al: Protein transduction assisted by polyethylenimine-cationized carrier proteins. J Biochem 137: 693-701, 2005.

29. Murata H, Sakaguchi M, Futami J, et al: Denatured and reversibly cationized p53 readily enters cells and simultaneously folds to the functional protein in the cells. Biochemistry 45: 6124-6132, 2006.

30. Futami M, Watanabe Y, Asama T, et al: Uniformly cationized protein efficiently reaches the cytosol of mammalian cells. Bioconjug Chem 23: 2025-2031, 2012.

31. Schilling D, Thomas K, Nixdorff K, Vogel SN and Fenton MJ: Toll-like receptor 4 and Toll-IL-1 receptor domain-containing adapter protein (TIRAP)/myeloid differentiation protein 88 adapter-like (Mal) contribute to maximal IL-6 expression in macrophages. J Immunol 169: 5874-5880, 2002.

32. Loiarro M, Sette C, Gallo G, et al: Peptide-mediated interference of TIR domain dimerization in MyD88 inhibits interleukin-1-dependent activation of NF-\{kappa\}B. J Biol Chem 280: 15809-15814, 2005.

33. Kaplan IM, Wadia JS and Dowdy SF: Cationic TAT peptide transduction domain enters cells by macropinocytosis. J Control Release 102: 247-253, 2005.

34. Bassi R, Giussani P, Anelli V, et al: HMGB1 as an autocrine stimulus in human T98G glioblastoma cells: role in cell growth and migration. J Neurooncol 87: 23-33, 2008.

35. Kataoka $\mathrm{K}$, Ono $\mathrm{T}$, Murata $\mathrm{H}$, et al: $\mathrm{S} 100 \mathrm{~A} 7$ promotes the migration and invasion of osteosarcoma cells via the receptor for advanced glycation end products. Oncol Lett 3: 1149-1153, 2012.

36. Hudson BI, Kalea AZ, Del Mar Arriero M, et al: Interaction of the RAGE cytoplasmic domain with diaphanous-1 is required for ligand-stimulated cellular migration through activation of Rac1 and Cdc42. J Biol Chem 283: 34457-34468, 2008.

37. Yamamoto K, Murata H, Putranto EW, et al: DOCK7 is a critical regulator of the RAGE-Cdc42 signaling axis that induces formation of dendritic pseudopodia in human cancer cells. Oncol Rep 29: 1073-1079, 2013.

38. Deane R, Singh I, Sagare AP, et al: A multimodal RAGE-specific inhibitor reduces amyloid $\beta$-mediated brain disorder in a mouse model of Alzheimer disease. J Clin Invest 122: 1377-1392, 2012.

39. Griffin WS, Sheng JG, McKenzie JE, et al: Life-long overexpression of S100beta in Down's syndrome: implications for Alzheimer pathogenesis. Neurobiol Aging 19: 401-405, 1998.

40. Mrak RE and Griffinbc WS: The role of activated astrocytes and of the neurotrophic cytokine S100B in the pathogenesis of Alzheimer's disease. Neurobiol Aging 22: 915-922, 2001. 